\title{
Structural features conserved in subclass of type II arabinogalactan
}

\author{
Kengo Ito ${ }^{1}$, Kurumi Fukuoka², Naho Nishigaki ${ }^{2}$, Katsuya Hara², \\ Yoshihisa Yoshimi ${ }^{2,3}$, Hiroaki Kuki ${ }^{2}$, Daisuke Takahashi ${ }^{2,4}$, Yoichi Tsumuraya ${ }^{2}$, \\ Toshihisa Kotake ${ }^{2,4, *}$ \\ ${ }^{1}$ Department of Biochemistry and Molecular Biology, Faculty of Science, Saitama University, 255 Shimo-okubo, Sakura-ku, \\ Saitama 338-8570, Japan; ${ }^{2}$ Division of Life Science, Graduate School of Science and Engineering, Saitama University, \\ 255 Shimo-okubo, Sakura-ku, Saitama 338-8570, Japan; ${ }^{3}$ Department of Biochemistry, University of Cambridge, Cambridge \\ CB2 1QW, UK; ${ }^{4}$ Green Biology Research Center, Saitama University, 255 Shimo-okubo, Sakura-ku, Saitama 338-8570, Japan \\ *E-mail: kotake@mail.saitama-u.ac.jp Tel: +81-48-858-3955Ｆax: +81-48-858-3384
}

Received May 25, 2020; accepted July 21, 2020 (Edited by T. Demura)

\begin{abstract}
Arabinogalactan-proteins (AGPs) are extracellular proteoglycans, which are presumed to participate in the regulation of cell shape, thus contributing to the excellent mechanical properties of plants. AGPs consist of a hydroxyprolinerich core-protein and large arabinogalactan (AG) sugar chains, called type II AGs. These AGs have a $\beta$-1,3-galactan backbone and $\beta$-1,6-galactan side chains, to which other sugars are attached. The structure of type II AG differs depending on source plant, tissue, and age. Type II AGs obtained from woody plants in large quantity as represented by gum arabic and larch $\mathrm{AG}$, here designated gum arabic-subclass, have a $\beta$-1,3;1,6-galactan structure in which the $\beta$-1,3-galactan backbone is highly substituted with short $\beta$-1,6-galactan side chains. On the other hand, it is unclear whether type II AGs found as the glycan part of AGPs from herbaceous plants, here designated AGP-subclass, also have conserved $\beta$-1,3:1,6-galactan structural features. In the present study we explore similarities of type II AG structures in the AGP-subclass. Type II AGs in fractions obtained from spinach, broccoli, bok choy, komatsuna, and cucumber were hydrolyzed into galactose and $\beta$-1,6-galactooligosaccharides by specific enzymes. Based on the proportion of these sugars, the substitution ratio of the $\beta$-1,3-galactan backbone was calculated as $46-58 \%$ in the five vegetables, which is consistently lower than what is seen in gum arabic and larch AG. Although most side chains were short, long chains such as $\beta$-1,6-galactohexaose chains were also observed in these vegetables. The results suggest a conserved $\beta-1,3 ; 1,6$-galactan structure in the AGP-subclass that distinguishes it from the gum arabic-subclass.
\end{abstract}

Key words: arabinogalactan-protein, enzymatic fragmentation, glycan structure, 6-galactan side chain, type II arabinogalactan, $\beta-1$.

The excellent mechanical properties of plants are provided by sophisticated tissue structures, in which cell shape is autonomously and dynamically regulated. It is well-known that the shape of plant cells is primarily determined by cellulose microfibrils in the cell walls, but the functions of other cell wall components in the regulation of cell shape are not fully understood.

Arabinogalactan-proteins (AGPs) are conserved extracellular proteoglycans consisting of a hydroxyproline-rich core-protein and a large portion of glycan moieties, called type II arabinogalactans (AGs) (Fincher et al. 1983; Majewska-Sawka and Nothnagel 2000; Seifert and Roberts 2007). As the Arabidopsis sos5/fla4 mutant with a defect in the core-protein gene exhibits a root swelling phenotype under salt stress, AGPs are presumed to participate in the regulation of cell shape (Shi et al. 2003). In suspension cultured tobacco cells, cell bulging is caused by treatment with $\beta$-Yariv reagent, a glycosyl-phenylazo compound specifically binding to type II AGs (Sardar et al. 2006). In addition, drastic in vivo degradation of type II AGs by expression of fungal glycoside hydrolase results in severe tissue disorganization in hypocotyls of Arabidopsis (Yoshimi et al. 2020). On the basis of previous studies, type II AGs are expected to be necessary for the functioning of AGPs. However, it is not yet clear in what way the glycan

Abbreviations: ABEE, $p$-aminobenzoic acid ethyl ester; AG, arabinogalactan; AGP, arabinogalactan-protein; $\alpha$-L-Arafase, $\alpha$-L-arabinofuranosidase from Aspergillus niger; Gal, galactose; $\mathrm{Gal}_{2}, \beta$-1,6-galactobiose; $\mathrm{Gal}_{3}, \beta$-1,6-galactotriose; $\mathrm{Gal}_{4}, \beta$-1,6-galactotetraose; $\mathrm{Gal}_{5}, \beta$-1,6-galactopentaose; $\mathrm{Gal}_{6}$, $\beta$-1,6-galactohexaose; GalA, galacturonic acid; HPLC, high performance-liquid chromatography; L-Ara, L-arabinose; MeGlcA, 4-O-methyl-glucuronic acid; MeGlcAGal, MeGlcA- $\beta$-1,6-Gal; rAnGlcAase, recombinant $\beta$-glucuronidase from Aspergillus niger; rIl3GAL, recombinant exo- $\beta$-1,3-galactanase from Irpex lacteus; rNcEn3GAL, recombinant endo- $\beta$-1,3-galactanase from Neurospora crassa.

This article can be found at http://www.jspcmb.jp/

Published online December 11, 2020 
structure is important for their physiological functions.

Plant AGs are classified into two groups, namely type I AG and type II AG. Whereas type I AG consists of $\beta$-1,4-galactan decorated with $\mathrm{L}$-arabinose (L-Ara), type II AG has $\beta$-1,3-galactan backbone with $\beta-1,6$ galactan side chains, called $\beta$-1,3;1,6-galactan, to which L-Ara, glucuronic acid (GlcA), 4-O-methyl-glucuronic acid (MeGlcA), and/or L-fucose are attached (Mohnen 2008; Tan et al. 2010; Tryfona et al. 2010, 2012). Since gum arabic and larch AG, belonging to the gum arabic-subclass according to the categorization used in this study, can be obtained in large quantity and are commercially available type II AGs, they are sometimes regarded as representative type II AGs. However, the structures of type II AGs are diverse not only in their non-reducing terminal residues, which include $\alpha$-Larabinofuranosyl and 4-O-methyl- $\beta$-glucuronosyl, but also in their basic $\beta$-1,3:1,6-galactan structure. Indeed, type II AGs found as glycan parts of AGPs, designated as AGP-subclass in this paper, likely have several features different from gum arabic-subclass AGs (Figure 1, Supplementary Figure S1). First, the substitution ratio of the $\beta$-1,3-galactan backbone in the AGP-subclass seems to be lower than the more than $90 \%$ estimated for gum arabic and larch AG based on previous reports (Akiyama et al. 1984; Inaba et al. 2015; Ponder and Richards 1997; Tryfona et al. 2012; Tsumuraya et al. 2019). Second, the degree of polymerization of $\beta$-1,6-galactosyl side chains, that is the length of galactose (Gal) side chains, may also differ between the two type II AG subclasses. Long side chains such as $\beta$-1,6-galactopentaose $\left(\mathrm{Gal}_{5}\right)$ and $\beta-1,6$ galactohexaose $\left(\mathrm{Gal}_{6}\right)$ side chains, which are not seen in gum arabic and larch AG, may be conserved in the AGP-subclass (Akiyama et al. 1984; Ponder and Richards 1997). These differences may reflect distinct physiological functions of the AGP-subclass and gum arabic-subclass in vivo. However, it has not been studied on a distinct conserved basic $\beta$-1,3;1,6-galactan structure in AGPsubclass focusing on side chain length and substitution ratio of backbone.

For the present study, we investigated the structure of AGP-subclass type II AGs from five vegetable tissues: leaves of spinach (Spinacia oleracea), flower buds of broccoli (Brassica oleracea var. italica), leaves of bok choy (Brassica rapa var. chinensis), leaves of komatsuna (Brassica rapa var. perviridis), and fruits of cucumber (Cucumis sativus L.) purchased from a local market. Bok choy and komatsuna are the same species but different cultivars. Broccoli is also a species belonging to the Brassica genus. These vegetables were homogenized in $0.1 \mathrm{M} \mathrm{NaCl}$ with a blender mixer. Soluble cell wall polysaccharides including AGPs were extracted as follows: (1) homogenates were boiled at $100^{\circ} \mathrm{C}$ for $20 \mathrm{~min}$ and then filtered; (2) after centrifugation at $5,000 \mathrm{~g}$ at $4^{\circ} \mathrm{C}$ for $10 \mathrm{~min}$, the supernatant was collected and
Type II AG AGP-subclass

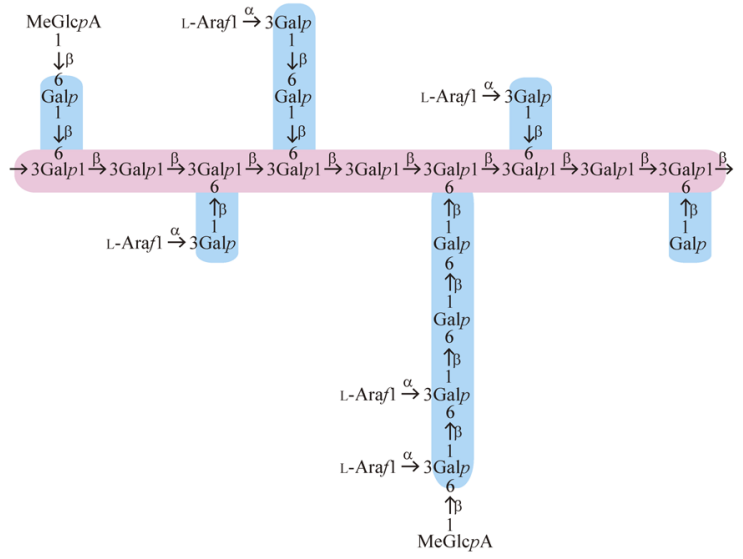

Type II AG gum arabic-subclass

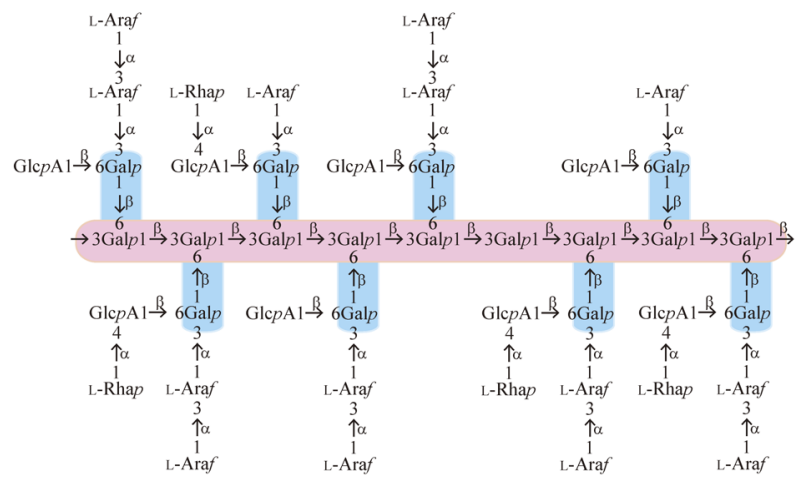

Figure 1. Structural difference between AGP-subclass and gum arabic-subclass. Model glycan structures for AGP-subclass and gum arabic AGs based on previous reports are shown (Akiyama et al. 1984; Kitazawa et al. 2013; Shimoda et al. 2014; Tsumuraya et al. 2019). The $\beta$-1,3-galactan backbone is shown in pink and $\beta$-1,6-galactan side chains in blue. The $f$ and $p$ indicate furanose and pyranose forms, respectively. Note that the structure for AGP-subclass does not represent all type II AGs found as glycan parts of AGPs, because there are variations in the length of $\beta$-1,6-galactan side chains and in terminal residues such as $\alpha$-L-arabinofuranosyl and 4-O-methyl- $\beta$-glucuronosyl residues. A model structure for larch AG is shown in Supplementary Figure S1.

concentrated by an evaporator. Polysaccharides in the supernatant were precipitated with $80 \%(\mathrm{v} / \mathrm{v})$ ethanol at $4^{\circ} \mathrm{C}$ for 1 day, collected by centrifugation at $5,000 \mathrm{~g}$ at $4^{\circ} \mathrm{C}$ for $10 \mathrm{~min}$, and dialyzed against water at $4^{\circ} \mathrm{C}$ for 2 days. The dialyzed polysaccharides were lyophilized and used as the crude polysaccharide (CP) fraction in the following experiments.

Prior to the structural analysis of type II AGs, sugar composition of polysaccharides contained in the $\mathrm{CP}$ fractions was determined. Polysaccharides in CP fractions were hydrolyzed into monosaccharides by $2 \mathrm{M}$ trifluoroacetic acid at $121^{\circ} \mathrm{C}$ for $60 \mathrm{~min}$, which were analyzed by high-performance anion-exchange chromatography with pulsed amperometric detection as described previously (Ishikawa et al. 2000). All CP fractions showed a relatively high proportion of L-Ara 
and Gal, indicating that the fractions were AGP-rich. On the other hand, CP fractions also appeared to include various other cell wall polysaccharides such as pectin and starch (Supplementary Table S1). The proportion of these other polysaccharides differed among CP fractions prepared from distinct species. For instance, CP fractions from spinach and cucumber had relatively higher proportions of galacturonic acid (GalA, probably derived from pectin), than the others, while in fractions from komatsuna the proportion was very low.

In general, it is necessary to purify polysaccharide for the analysis of glycan structures. However, the purification process has been recognized as an obstacle for structural analysis because it is time-consuming work (Tsumuraya et al. 1988, 2019). To get structural information on type II AGs more easily, we subjected $\mathrm{CP}$ fractions to specific enzymatic fragmentation using a combination of fungal enzymes (Supplementary Figure S2). CP fractions were first digested with two specific galactanases, recombinant exo- $\beta$-1,3-galactanase from Irpex lacteus (rIl3GAL) and recombinant endo- $\beta-1,3-$ galactanase from Neurospora crassa (rNcEn3GAL) in $50 \mathrm{mM}$ acetate buffer ( $\mathrm{pH} 4.6$ ) at $37^{\circ} \mathrm{C}$ for $4 \mathrm{~h}$ (Kotake et al. 2009; Yoshimi et al. 2017). The hydrolysis of $\beta$-1,3-galactan backbone was confirmed by radial gel diffusion assay with $\beta$-Yariv reagent (Supplementary Figure S3) (Kitazawa et al. 2013; van Holst and Clarke 1985). The resulting Gal and oligosaccharides were coupled at their reducing terminals with $p$-aminobenzoic acid ethyl ester (ABEE) as described (Matsuura and Imaoka 1988; Tsumuraya et al. 2019). To remove nonreducing terminal $\alpha$-L-arabinofuranosyl and $4-O$ methyl- $\beta$-glucuronosyl residues attached to $\beta-1,6$ galactooligosaccharides, ABEE-labelled saccharides were further digested with $\alpha$-L-arabinofuranosidase $(\alpha$-L-Arafase) from Aspergillus niger (Megazyme), and recombinant $\beta$-glucuronidase from Aspergillus niger (rAnGlcAase) in $50 \mathrm{mM}$ acetate buffer $(\mathrm{pH} 4.6)$ at $37^{\circ} \mathrm{C}$ for $12 \mathrm{~h}$ (Konishi et al. 2008; Yoshimi et al. 2020), and then analyzed on an HPLC system equipped with a TSKgel Amide- 80 column $(4.6 \times 250 \mathrm{~mm}$; Tosoh, Tokyo, Japan) in accordance with a previous study (Tsumuraya et al. 2019). Here, Gal is derived from $\beta$-galactosyl residues of the $\beta$-1,3-galactan backbone where there is no side chain attached, while $\beta$-1,6-galactooligosaccharides are those having side chains. It should be noted that a $\beta$-1,6-galactan side chain converted to $\beta-1,6-$ galactooligosaccharide in the above steps will always have one additional Gal residue derived from the backbone, compared to the side chain itself. For example, a $\beta-1,6-$ galactobiose $\left(\mathrm{Gal}_{2}\right)$ side chain attached to the backbone is turned into $\beta$-1,6-galactotriose $\left(\mathrm{Gal}_{3}\right)$ in resultant fractions after enzymatic digestion (Supplementary Figure S2).

ABEE-labelled Gal and $\beta$-1,6-galactooligosaccharides

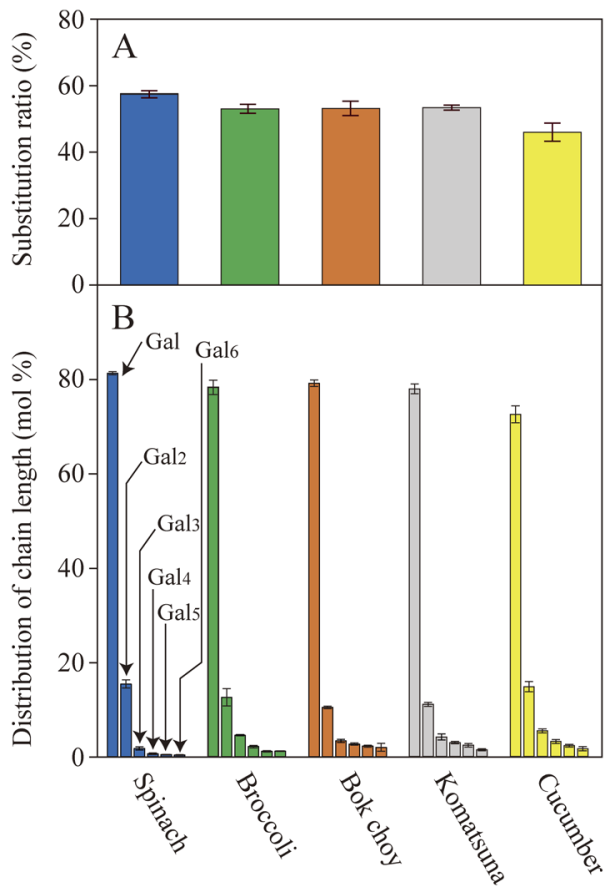

Figure 2. Structural features of $\beta$-1,3:1,6-galactan as found in the AGP-subclass. The substitution ratio of $\beta$-1,3-galactan backbones by $\beta$-1,6-galactan side chains in five vegetables is shown (A). The ratio was calculated based on the proportion of Gal (residue without branching in $\beta$-1,3-galactan) and $\beta$-1,6-galactooligosaccharides (branching residues in $\beta$-1,3-galactan). The distribution of the Gal length of side chains was also calculated (B). The values shown in mol \% for Gal, $\mathrm{Gal}_{2}, \mathrm{Gal}_{3}, \mathrm{Gal}_{4}, \mathrm{Gal}_{5}$, and $\mathrm{Gal}_{6}$ of side chains were calculated based on the peak area of ABEE-labelled $\mathrm{Gal}_{2}, \mathrm{Gal}_{3}, \mathrm{Gal}_{4}, \mathrm{Gal}_{5}, \mathrm{Gal}_{6}$, and $\mathrm{Gal}_{7}$, respectively. Data are mean values with $\pm S D$ ( $n=3$ biological replicates). Representative chromatograms are shown in Supplementary Figure S4.

were clearly separated by HPLC (Supplementary Figure S4), which enabled us to determine the ratios of these saccharides. As only reducing terminal residues are labelled by ABEE (Supplementary Figure S2), the peak area reflects the mol concentration of each saccharide. Based on the ratio between Gal and the sum of $\beta-1,6$ galactooligosaccharides, the substitution ratio of the $\beta$-1,3-galactan backbone can be calculated. As shown in Figure 2A, the ratio was high in type II AGs from spinach (58\%) in contrast to komatsuna (46\%). But these values were clearly lower than those ( $>90 \%$ ) of gum arabic and larch AG estimated based on previous reports (Akiyama et al 1984; Ponder and Richards 1997). Subsequently, the distribution of the Gal length of side chains was also calculated. The distribution of side chain length in type II AGs was similar in the five vegetables. Although side chains consisting of single Gal residues accounted for more than $70 \%$ of the total side chain amount, side chains longer than $\beta$-1,6-galactotetraose $\left(\mathrm{Gal}_{4}\right)$ were observed in all vegetables (Figure $2 \mathrm{~B}$ ). Because of the limitations of HPLC analysis, very long $\beta-1,6$ galactooligosaccharides were not detected. However, it is highly probable that type II AGs from these vegetables 
include side chains longer than $\mathrm{Gal}_{6}$ as seen in type II AGs from European pear (Tsumuraya et al. 2019).

The present analysis quickly and easily gives structural information on $\beta-1,3: 1,6$-galactan basic structure. On the other hand, because there might be side chains decorated with $\alpha$-L-fucosyl and $\beta$-L-arabinopyranosyl residues that are not converted to $\beta$-1,6-galactooligosaccharides by the combination of enzymes used here, some of the side chains could not be analyzed in the present study. However, as these side chains are not abundant, the results obtained in the present study would not be largely affected. In fact, such hypothetical unknown oligosaccharides were hardly detected in HPLC chromatograms (Supplementary Figure S4).

This study suggests that the AGP-subclass AGs found in five vegetables have a conserved basic $\beta-1,3: 1,6$ galactan structure and the substitution ratios of their $\beta$-1,3-galactan backbones and the distributions of the Gal length of side chains are different from gum arabic and larch AG. On the other hand, to confirm the presumably conserved basic structure for the gum arabic-subclass, structures of type II AGs from other woody plant species should be analyzed. Different $\beta$-1,3:1,6-galactan structures probably arise from different properties of glycosyltransferases (GT) catalyzing the synthesis of $\beta$-1,3:1,6-galactan. One $\beta$-1,6-galactosyltransferase creating branches on the $\beta$-1,3-galactan backbone and another $\beta$-1,6-galactosyltransferase catalyzing chain elongation of $\beta$-1,6-galactan probably determine the substitution ratio and distribution of the Gal length of side chains, respectively (Dilokpimol et al 2014; Geshi et al. 2013). In future studies, through the characterization of these GTs, the mechanism for the synthesis of the conserved $\beta$-1,3:1,6-galactan basic structures in the AGPsubclass should be clarified.

\section{Acknowledgements}

This work was supported by MEXT KAKENHI Grant-in-Aid for Scientific Research on Innovative Areas "Plant-Structure Optimization Strategy" to T. Kotake [no. 18H05495] and Grant-inAid for Scientific Research to T. Kotake [no. 19K06702] and to D. Takahashi [no. 20K15494].

\section{Author contributions}

K.I., Y.Y., H.K., Y.T., and T.K. designed research; K.I., K.F., N.N., K.H., Y.Y. and T.K. performed research; K.I., N.N., K.H., Y.Y., and T.K. analyzed data; K.I., H.K., D.T., Y.T., and T.K. wrote the paper.

\section{References}

Akiyama Y, Eda S, Kato K (1984) Gum arabic is a kind of arabinogalactan-protein. Agric Biol Chem 48: 235-237

Dilokpimol A, Poulsen CP, Vereb G, Kaneko S, Schulz A, Geshi N (2014) Galactosyltransferases from Arabidopsis thaliana in the biosynthesis of type II arabinogalactan: Molecular interaction enhances enzyme activity. BMC Plant Biol 14: 90
Fincher GB, Stone BA, Clarke AE (1983) Arabinogalactan-proteins: Structure, biosynthesis, and function. Annu Rev Plant Physiol 34: 47-70

Geshi N, Johansen JN, Dilokpimol A, Rolland A, Belcram K, Verger S, Kotake T, Tsumuraya Y, Kaneko S, Tryfona T, et al. (2013) A galactosyltransferase acting on arabinogalactan protein glycans is essential for embryo development in Arabidopsis. Plant $J$ 76: $128-137$

Inaba M, Maruyama T, Yoshimi Y, Kotake T, Matsuoka K, Koyama T, Tryfona T, Dupree P, Tsumuraya Y (2015) L-Fucose-containing arabinogalactan-protein in radish leaves. Carbohydr Res 415: $1-11$

Ishikawa M, Kuroyama H, Takeuchi Y, Tsumuraya Y (2000) Characterization of pectin methyltransferase from soybean hypocotyls. Planta 210: 782-791

Kitazawa K, Tryfona T, Yoshimi Y, Hayashi Y, Kawauchi S, Antonov L, Tanaka H, Takahashi T, Kaneko S, Dupree P, et al. (2013) $\beta$-Galactosyl Yariv reagent binds to the $\beta$-1,3-galactan of arabinogalactan proteins. Plant Physiol 161: 1117-1126

Konishi T, Kotake T, Soraya D, Matsuoka K, Koyama T, Kaneko S, Igarashi K, Samejima M, Tsumuraya Y (2008) Properties of family $79 \beta$-glucuronidases that hydrolyze $\beta$-glucuronosyl and 4-O-methyl- $\beta$-glucuronosyl residues of arabinogalactan-protein. Carbohydr Res 343: 1191-1201

Kotake T, Kitazawa K, Takata R, Okabe K, Ichinose H, Kaneko S, Tsumuraya Y (2009) Molecular cloning and expression in Pichia pastoris of a Irpex lacteus exo- $\beta-(1 \rightarrow 3)$-galactanase gene. Biosci Biotechnol Biochem 73: 2303-2309

Majewska-Sawka A, Nothnagel EA (2000) The multiple roles of arabinogalactan proteins in plant development. Plant Physiol 122: 3-9

Matsuura F, Imaoka A (1988) Chromatographic separation of asparagine-linked oligosaccharides labeled with an ultravioletabsorbing compound, $p$-aminobenzoic acid ethyl ester. Glycoconj J 5: 13-26

Mohnen D (2008) Pectin structure and biosynthesis. Curr Opin Plant Biol 11: 266-277

Ponder GR, Richards GN (1997) Arabinogalactan from western larch, Part III: Alkaline degradation revisited, with novel conclusions on molecular structure. Carbohydr Polym 34: 251-261

Sardar HS, Yang J, Showalter AM (2006) Molecular interactions of arabinogalactan proteins with cortical microtubules and F-actin in Bright Yellow-2 tobacco cultured cells. Plant Physiol 142: 1469-1479

Seifert GJ, Roberts K (2007) The biology of arabinogalactan proteins. Annu Rev Plant Biol 58: 137-161

Shi H, Kim Y, Guo Y, Stevenson B, Zhu JK (2003) The Arabidopsis SOS5 locus encodes a putative cell surface adhesion protein and is required for normal cell expansion. Plant Cell 15: 19-32

Shimoda R, Okabe K, Kotake T, Matsuoka K, Koyama T, Tryfona T, Liang H-C, Dupree P, Tsumuraya Y (2014) Enzymatic fragmentation of carbohydrate moieties of radish arabinogalactan-protein and elucidation of the structures. Biosci Biotechnol Biochem 78: 818-831

Tan L, Varnai P, Lamport DT, Yuan C, Xu J, Qiu F, Kieliszewski MJ (2010) Plant $O$-hydroxyproline arabinogalactans are composed of repeating trigalactosyl subunits with short bifurcated side chains. J Biol Chem 285: 24575-24583

Tryfona T, Liang H-C, Kotake T, Kaneko S, Marsh J, Ichinose H, Lovegrove A, Tsumuraya Y, Shewry PR, Stephens E, et al. (2010) Carbohydrate structural analysis of wheat flour arabinogalactan 
protein. Carbohydr Res 345: 2648-2656

Tryfona T, Liang H-C, Kotake T, Tsumuraya Y, Stephens E, Dupree P (2012) Structural characterization of Arabidopsis leaf arabinogalactan polysaccharides. Plant Physiol 160: 653-666

Tsumuraya Y, Ogura K, Hashimoto Y, Mukoyama H, Yamamoto S (1988) Arabinogalactan-proteins from primary and mature roots of radish (Raphanus sativus L.). Plant Physiol 86: 155-160

Tsumuraya Y, Ozeki E, Ooki Y, Yoshimi Y, Hashizume K, Kotake T (2019) Properties of arabinogalactan-proteins in European pear (Pyrus communis L.) fruits. Carbohydr Res 485: 107816

van Holst GJ, Clarke AE (1985) Quantification of arabinogalactan- protein in plant extracts by single radial gel diffusion. Anal Biochem 148: 446-450

Yoshimi Y, Hara K, Yoshimura M, Tanaka N, Higaki T, Tsumuraya Y, Kotake T (2020) Expression of a fungal exo- $\beta$-1,3-galactanase in Arabidopsis reveals a role 'of' type II arabinogalactan in the regulation of cell shape. J Exp Bot 71: 5414-5424

Yoshimi Y, Yaguchi K, Kaneko S, Tsumuraya Y, Kotake T (2017) Properties of two fungal endo- $\beta$-1,3-galactanases and their synergistic action with an exo- $\beta-1,3$-galactanase in degrading arabinogalactan-proteins. Carbohydr Res 453-454: 26-35 